\title{
P155: Technologies to measure hand hygiene: examining the incorporation of the World Health Organisation (WHO) 5 moments
}

C Dawson

From 2nd International Conference on Prevention and Infection Control (ICPIC 2013)

Geneva, Switzerland. 25-28 June 2013

\section{Introduction}

A case study of Hand Hygiene (HH) auditing within a UK NHS Acute Hospital resulted in the exploration of technology to improve efficiency and accuracy of $\mathrm{HH}$ measurement as per the evidence-based WHO 5 Moments of Hand Hygiene.

\section{Objectives}

1. Determine the current state of measurement at case study site and identify perceived strengths/weaknesses.

2. Use WHO 5 Moments as benchmark to evaluate electronic monitoring (EM) technologies designed to capture $\mathrm{HH}$ compliance, and assess their perceived feasibility for use at case study site.

\section{Methods}

Data was collected over 15 months. Observation and qualitative interviewing were used to produce a current state map of measurement. A structured literature review assessed Fit-For-Purpose of EM technologies. The current state map was used to assess their feasibility for use at case study site.

\section{Results}

From a target pool of 124, 45 Healthcare Professionals (HP) were recruited including Infection Control, Nurses and Consultants; all involved in $\mathrm{HH}$ auditing; collecting data, receiving feedback or being subject to observation.

1. No explicit reference to WHO 5 Moments was included in current measurement of manual auditing using direct observation based upon standards for $\mathrm{HH}$ Technique (How) and Process (When).
Lack of clarity and consistency in content of audit feedback (AF) and the feedback process was identified as a weakness by all 30 participants (pp) interviewed.

A second weakness was lack of synergy between training content and content of AF data; training incorporates WHO 5 Moments yet AF contains no reference to performance at these times.

These weaknesses led the majority of pp $(22 / 30)$ to conclude AF data is often 'meaningless'.

2. No technologies able to monitor, measure and provide feedback on all 5 WHO Moments; 1, 4, 5 show potential for technology monitoring. HP are open to technology; all 13 ward based pp interested in potential for personal development and group improvement. Concerns raised in all interviews included 'Big Brother' culture and lack of Fit-for-Purpose tools.

\section{Conclusion}

Weaknesses in current $\mathrm{HH}$ auditing processes can lead to perceptions of 'meaningless' data. No EM system currently fully supports the WHO 5 Moments, and whilst $\mathrm{HP}$ are open to technology, perceptions regarding use still exist and require addressing if EM systems are to be considered alternatives to direct observation.

\section{Disclosure of interest}

None declared.

Published: 20 June 2013 